\title{
A method for predicting geometric characteristics of polymer deposition during fused-filament-fabrication.
}

\author{
Michael Hebda $^{1}$, Claire McIlroy ${ }^{2}$, Ben Whiteside ${ }^{1}$, Fin Caton-Rose $^{1}$, Phil Coates $^{1}$ \\ ${ }^{1}$ Faculty of Engineering and Informatics, University of Bradford \\ ${ }^{2}$ School of Mathematical Sciences, University of Nottingham
}

\begin{abstract}
In recent years 3D printing has gained popularity amongst industry professionals and hobbyists alike, with many new types of Fused Filament Fabrication (FFF) apparatus types becoming available on the market. A massively overlooked component of FFF is the requirement for a simple method to calculate the geometries of polymer depositions extruded during the FFF process. Manufacturers have so far achieved adequate methods to calculate tool-paths through so called slicer software packages which calculate the required velocities of extrusion from prior knowledge and data. Presented here is a method for obtaining a series of equations for predicting height, width and cross-sectional area values for given processing parameters within the FFF process for initial laydown on to a glass surface.
\end{abstract}

Keywords:

Fused Filament Fabrication, Conservation of Mass, Cross-sectional Geometry
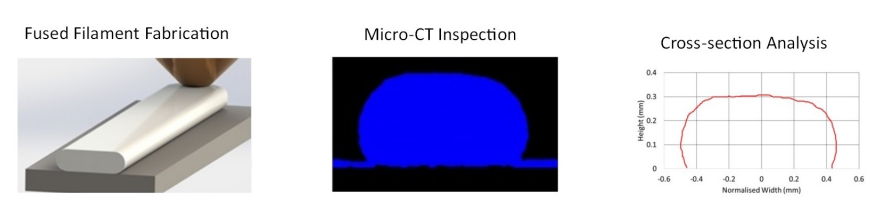

Model Prediction
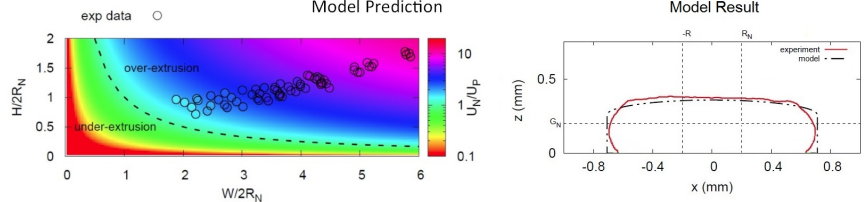

Figure 1: Visual Abstract

\section{Introduction}

Fused filament fabrication (FFF), a form of Additive Manufacture (AM), is in common use in many research and industrial areas, mainly for its prototyping functionalities. This method of prototyping is carried out by extruding filaments of polymer material onto a build plate in order to build up a $3 \mathrm{D}$ object layer-by-layer $[1,2,3,4]$.The material used is a thermoplastic polymer, which is fed as a solid filament into a heated nozzle where it melts and flows as a polymer melt onto the preceding layer. Here it cools rapidly and solidifies to form the new solid layer. The deposition method is typically a continuous deposited filament which is generated by traversing the nozzle around the build stage as material is extruded.

The influence of the temperature, flow, material properties and build strategy on the quality of the final product

\begin{tabular}{|l|l|}
\hline$G_{N}$ & Nozzle set gap height \\
\hline$U_{P}$ & $\begin{array}{l}\text { Extruder travel velocity in relation to the } \\
\text { build area }\end{array}$ \\
\hline$U_{N}$ & Radially averaged nozzle exit velocity \\
\hline$U_{0}$ & Filament feed velocity \\
\hline$R_{N}$ & Nozzle exit radius \\
\hline$R_{0}$ & Fed filament radius \\
\hline$A_{P}$ & $\begin{array}{l}\text { Predicted cross-sectional area of deposited fil- } \\
\text { ament }\end{array}$ \\
\hline$A_{N}$ & $\begin{array}{l}\text { Resultant cross-sectional area of deposited fil- } \\
\text { ament }\end{array}$ \\
\hline$A_{e x p}$ & $\begin{array}{l}\text { Area of experimental polymer deposition } \\
\text { cross-section }\end{array}$ \\
\hline$H$ & Polymer deposition height \\
\hline$W$ & Polymer deposition width \\
\hline$\alpha$ & pre-factor \\
\hline
\end{tabular}

Table 1: Table showing a list of mathematical symbols used.

is of significant interest. Many articles have been published that study build strategies and the resulting mechanical properties $[5,6,7,8,9,10]$. There is much less research, however, dedicated to understanding how the balance of extrusion parameters (extrusion velocity $U_{N}$, transverse nozzle velocity $U_{P}$ and nozzle gap heights $G_{N}$ ) influences the deposited filament geometry.

Since tool paths are often directly calculated from 3D CAD data, there is little freedom in parameter control. A poor balance of these extrusion parameters can cause flaws within layer adhesion and lead to voids in the printed 
structure $[11,12]$; voids between lines of extruded polymer can have adverse effects on mechanical properties within FFF components $[6,11,13,14]$.

There have been a number of modelling approaches to capture the deposition process; a review can be found by Turner et al. [15]. In particular Agarwala et al. [16] and Bellini et al. [17] approximate feed rates $U_{0}$ required for a particular filament width $W$ and height $H$. Yardimci et al. [18] and Venkataraman et al. [19] have also explored the filament buckling mechanism that limits the feed rate. Spreading of the deposited filament has also been investigated by Crockett et al. [20], and a 2D simulation of this spreading process is presented by Bellini et al. [21]. However, it is noted by Turner et al. that 'there has been a limited degree of experimental validation of process models.'

More recently experimental comparisons have been presented by Gleadall et al. [22], who employ a new computationa efficient method based on conservation of volume to predict the geometry of a 3D lattice, and Comminal et al. [23], who employ a full (isothermal) computational fluid dynamics (CFD) model of a single deposited filament. Extrusion of single polymer filaments has many applications including antenna manufacture with conductive filaments [24], custom polymer vascular inserts [25] as well as scaffolding structures [26] for the initial layers of the FFF process.

Of particular interest to the AM community is a simplified model, which removes the need for knowledge of complex flow dynamics required by CFD simulations, that allows FFF users to quickly and efficiently test the effect of extrusion parameters on part geometry. CFD simulations, whilst having advanced in recent years in terms of efficiency are still very time consuming and require significant knowledge and training in comparison to a simplified mathematical model [27].

In particular, Comminal et al. [23] compare their full CFD model to a simplified model based on conservation of mass. Although this model is able to capture spreading of the filament in the $x y$-plane, this work does not explore the phenomenon of the actual filament height, $H$, extending above the nozzle gap size, $G_{N}$ in the $z$-direction. This is a common effect observed in FFF printing [21] and causes complications when subsequent filaments of molten polymer are extruded.

In this paper, we investigate the influence of extrusion parameters on track dimensions for an extended range of print speed ratios $\left(U_{N} / U_{P}\right)$ and nozzle gap sizes $G_{N}$ using CT scanning. We present a idealised model based on conservation of mass (similar to Ref. [23]), which is able to account for the filament height expanding above the nozzle gap size $\left(H>G_{N}\right)$ unlike previous studies. We compare two printing materials; ABS, an amorphous polymer melt containing rubber nanaoparticles, and PLA, a semicrystalline polymer melt. Volume changes during cystallization can often cause contraction of a melt upon cooling [28], thus ABS and PLA geometry is expected to differ sig- nificantly. Finally, we discuss the effect that changing the bed temperature has on filament spreading.

\section{Method}

In order to study the geometry of molten polymer material being deposited onto a build plate, an experiment was developed by which glass slides were mounted onto the bed of an FFF machine. Lines of extrudate were deposited onto the slide surface using a range of extrusion parameters. Depositing the polymer onto glass slides represents the deposition of the initial layer of the FFF process and also provides a sufficiently large enough difference in the density of materials to give a clear contrast during the subsequent CT scanning process.

\subsection{FFF Apparatus}

The extrusion temperature was kept at a $220^{\circ} \mathrm{C}$ with the platform surface being kept at $50^{\circ} \mathrm{C}$ with the experiments later being repeated at a platform temperature of $60^{\circ} \mathrm{C}$ to measure for differences due to thermal effects. The extrusion of the test material was carried out using a MakerBot Replicator 2 FFF machine for all samples in this experiment. The apparatus has a single nozzle extrusion system mounted on a cartesian axis setup controlled by stepper motors. Polymer filaments were mounted on a spool and processed through a gear and pinch wheel system into a heated nozzle of diameter $0.4 \mathrm{~mm}$ and extruded onto the heated platform. Both RS Components Natural PLA filament (SN: 832-0210) and RS Components White ABS filament (SN: 832-0315) was used, supplied by RS Components. The filament was $1.75 \mathrm{~mm}$ in diameter and was prepared fresh from the packet to avoid the effect of moisture and checked for consistent diameter using a pair of digital callipers. The nozzle used in conjunction with the extruder setup was a standard MK8 nozzle supplied with the MakerBot Replicator 2.

Thermo Scientific Menzel-Gläser slides were used, supplied by Agar Scientific. These were $1.0 \mathrm{~mm}$ thick, plain glass slides with dimensions of $76 \mathrm{~mm}$ x $26 \mathrm{~mm}$. Glass slides were chosen due to their similarity to glass build plates used in conjunction with FFF machines. In order to ensure the glass slides were mounted on a level printing surface, a laser measuring system was used by mounting a laser sensor onto the extruder mounting plate of the FFF equipment. The laser detector used was a Micro Epsilon optoNCDT 1401 in conjunction with Tenma power supply $72-8700 \mathrm{~A}$ run at $12 \mathrm{~V}$. The output signal of the laser was recorded using a Pico Technology PicoScope for Mixed Signal Processing and analysed using PicoScope 6 software. Replicator G software was used in order to control the FFF apparatus due to its simplicity and ability to send individual toolpath codes to the apparatus. In order to create a suitable toolpath code, toolpaths were studied from various software manufacturers as part of initial research. 


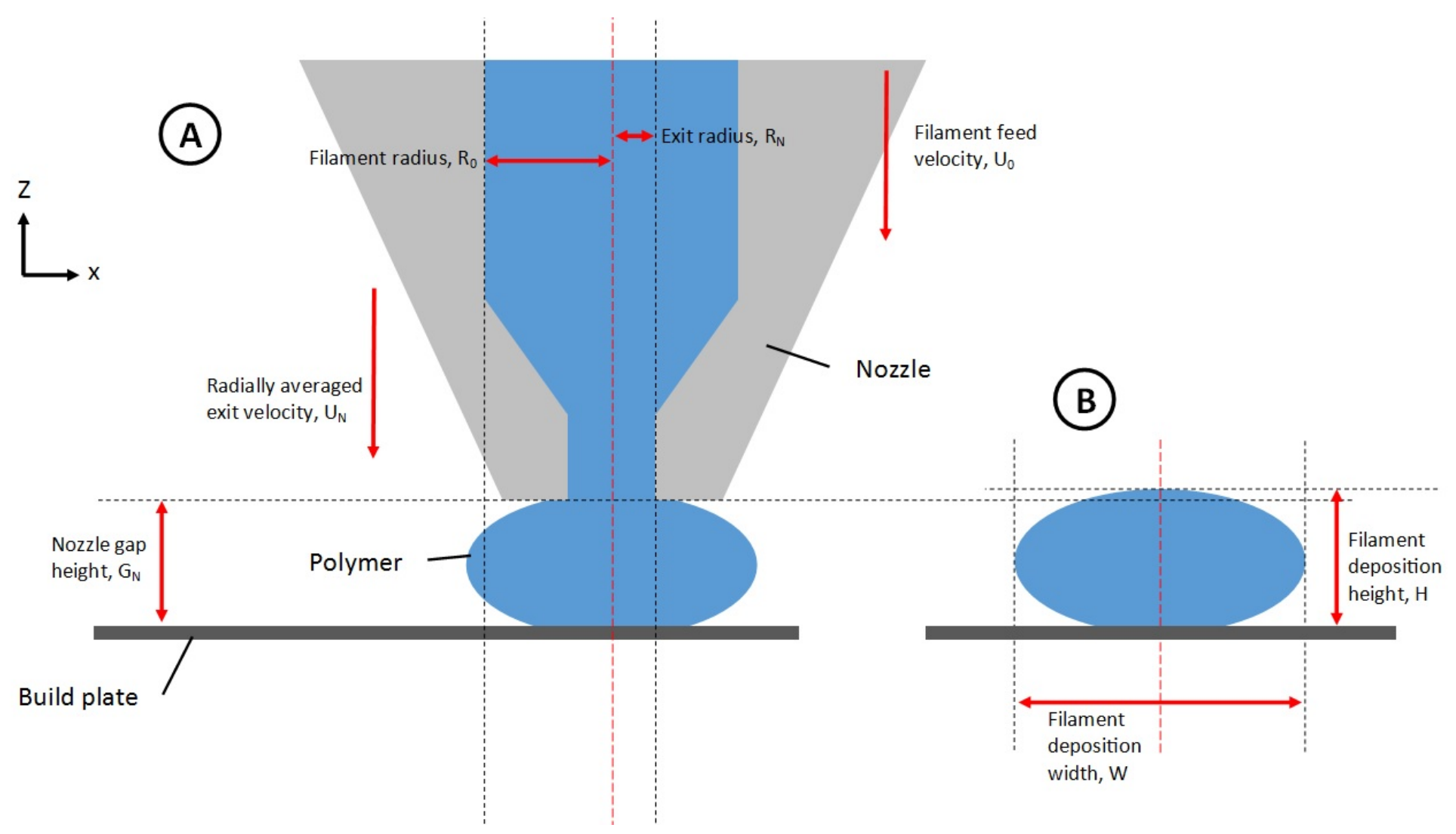

Figure 2: A schematic representation of the FFF process with the nozzle moving towards the viewer. (A): Initial deposition. (B): Steady state deposition, post-expansion.

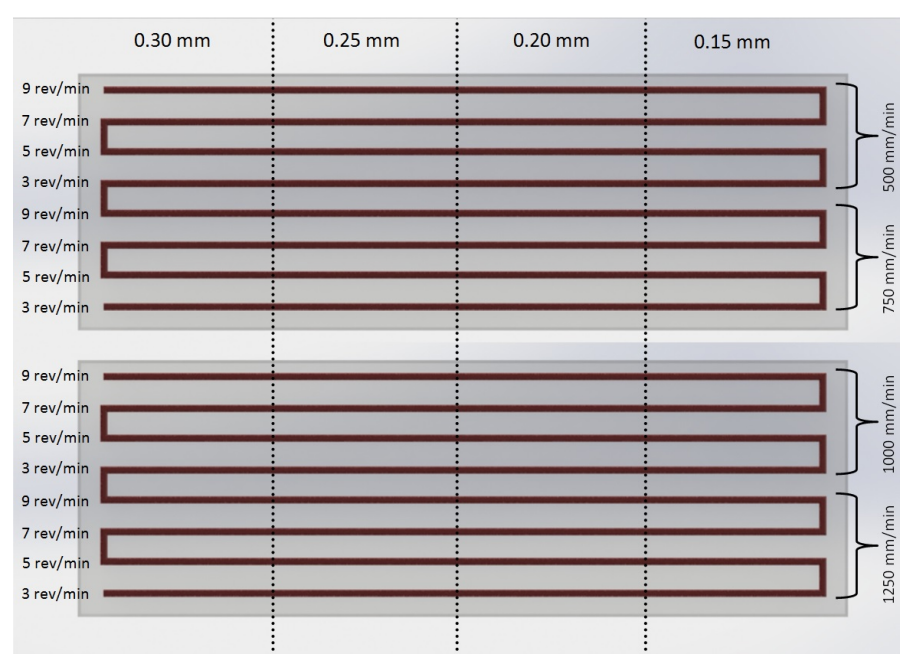

Figure 3: Visual layout of polymer deposition samples on two glass slides with nozzle gap heights $\left(G_{N}\right)$, filament feed velocities $\left(U_{0}\right)$ and build plate velocities $\left(U_{P}\right)$

The feed rates of 3 to $9 \mathrm{rev} / \mathrm{min}$ used for the extrusion velocities and represents both the lowest and highest velocities at which filament can be practically extruded without any adverse effects from pushing the apparatus to the upper and lower limits of extrusion. At the upper limit of extrusion the pinch wheel system would not be able to ap-

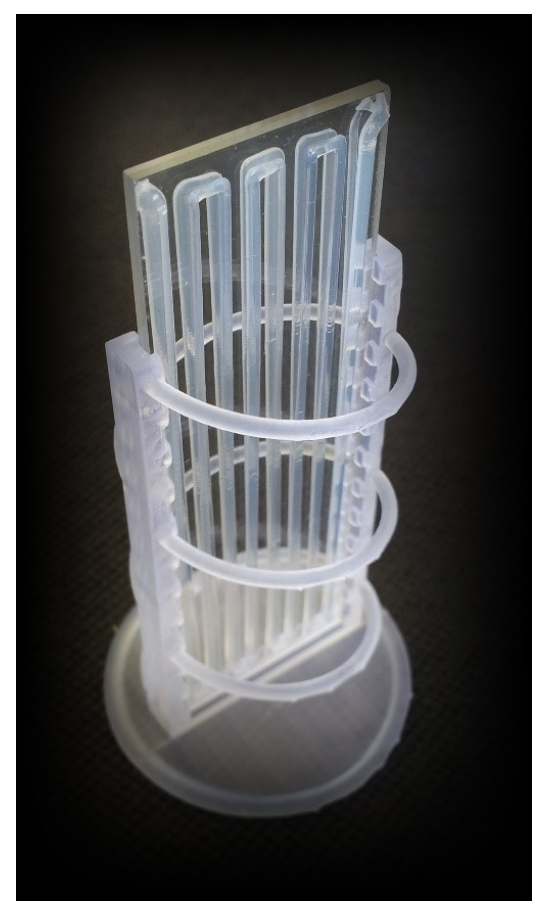

Figure 4: The CT stage used to mount both slides of samples back to back, PLA is the specific material used in this example. 
ply enough pressure to force material through the die [29], at the lower end the stepper motors used would not be able to turn in such minute increments causing incorrect feeding of the material, this in turn would affect the flow of molten polymer. The filament feed velocities, $U_{0}$, and resultant radially averaged exit velocities, $U_{N}$, are shown in table 2. All symbols used within this paper are shown in table 1.

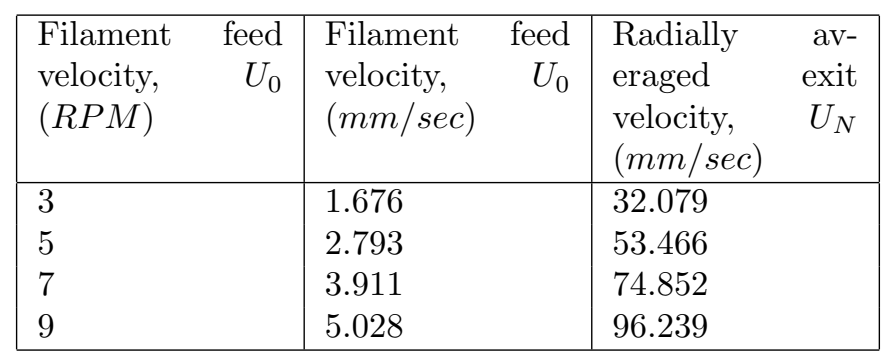

Table 2: Conversion table relating filament feed velocity, $U_{0}$, and radially averaged exit velocity, $U_{N}$.

Assuming a constant volume flux and density, the feed rate velocity is converted to an extrusion velocity via,

$$
U_{N}=U_{0}\left(\frac{R_{0}}{R_{N}}\right)^{2}
$$

The set layer thickness is specified using nozzle gap height $\left(G_{N}\right)$, which is the vertical distance between the tip of the nozzle and the top of the preceding layer. For this study, four nozzle heights were selected $(0.15 \mathrm{~mm}, 0.20$ $\mathrm{mm}, 0.25 \mathrm{~mm}$ and $0.30 \mathrm{~mm}$ ), representing a typical range of working nozzle heights for the chosen apparatus. The nozzle heights were used in conjunction with four travel velocities, $U_{P}$, four extrusion velocities, $U_{N}$. This gives a total of 64 samples over two slides; the layout patterns can be seen in Fig. 3. This method allowed the two slides to be mounted back to back and imaged in the CT apparatus in a single scan, as shown in Fig. 4.

\subsection{CT Scanning Apparatus}

The CT aparatus used was a Nikon XT H 225 allpurpose X-ray and CT inspection machine in conjunction with proprietary software and VGStudio MAX 2.2.4 software for visualisation and exportation of image stacks.

All CT scans of the samples were turned into image stacks at $0.1 \mathrm{~mm}$ intervals down the sample deposition. This process gave a pixel accuracy of $10 \mu \mathrm{m}$. A LabVIEW script was then created in order to process the image stacks in the following steps:

- Extract 2D section from CT scan

- Flatten image to glass slide surface baseline

- Apply labview contour algorithm

- Retrieve the height, width and cross-sectional area of the contour, bounded by the glass slide surface

- Export coordinate data as a .csv file

An example of an image processed using this LabVIEW script can be found in Fig. 5 .
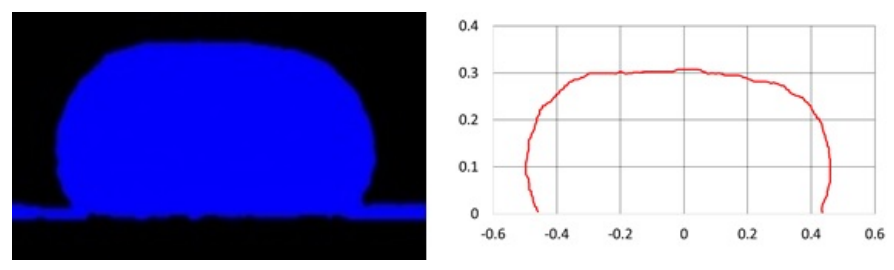

Figure 5: A polymer deposition cross-section taken from Micro-CT imaging (left) alongside its respective contour graph (right, measurements in millimetres), sample " $500 \mathrm{~mm} / \mathrm{min}-3 \mathrm{rev} / \mathrm{min}-0.30$ $\mathrm{mm} "$.

\section{Results}

In this section we show the results for PLA with build plate temperature $50{ }^{\circ} \mathrm{C}$. The comparison to ABS and different build plate temperatures is deferred to the Discussion section.

Height, width and cross-sectional area was measured from the CT images for each sample. For all samples, averages taken from 5 points in the centre of each sample's deposition were used in order to calculate all quantitative data, apart from the contour maps, these were taken from a central individual slice.

\subsection{Cross-sectional profiles}

Fig. 6 displays the contour outlines of all PLA samples with $50^{\circ} \mathrm{C}$ platform temperature grouped by extrusion velocity, $U_{N}$, and travel velocity, $U_{P}$, but with differing set nozzle gap heights, $G_{N}$, in each graph.

For all samples we can see that the height of each filament deposition is greater than the nozzle gap height prescribed by the apparatus i.e. $H>G_{N}$. This is due to the effect of the build plate and surrounding extrudate restricting the flow of material and confining the extruded filament to a finite space $[28,30]$, which occurs due to the downward force required to extrude the material exceeding the force which can be delivered by the feed system. Thus, the shape of the cross-section differs considerably to those shown in Ref. [23], where the deposited filament height is close to the nozzle gap size i.e. $H \approx G_{N}$.

Deposition height is both larger than the gap size and the width is greater than the nozzle diameter. Thus, we observe expansion in two directions, which is in contrast to assumptions, where polymer depositions are assumed to be either the width of the nozzle internal diameter (twice $R_{N}$ ), with the volume output being fixed through trial and error to achieve the correct deposition height, or the inverse where the set nozzle gap height is the assumed fixed factor $[3,31,32]$ and the volume output adjusted to acquire the correct deposition width.

Other visual observations of Fig. 6 show that the nozzle confines the filament extrusion and forms flattened geometries, this is particularly noticeable in the $0.15 \mathrm{~mm}-$ $3 \mathrm{rev} / \mathrm{min}$ samples which display flattened top surfaces. Larger cross-sections also display a more rounded shape with some having a more circular rather than elliptical geometry. 

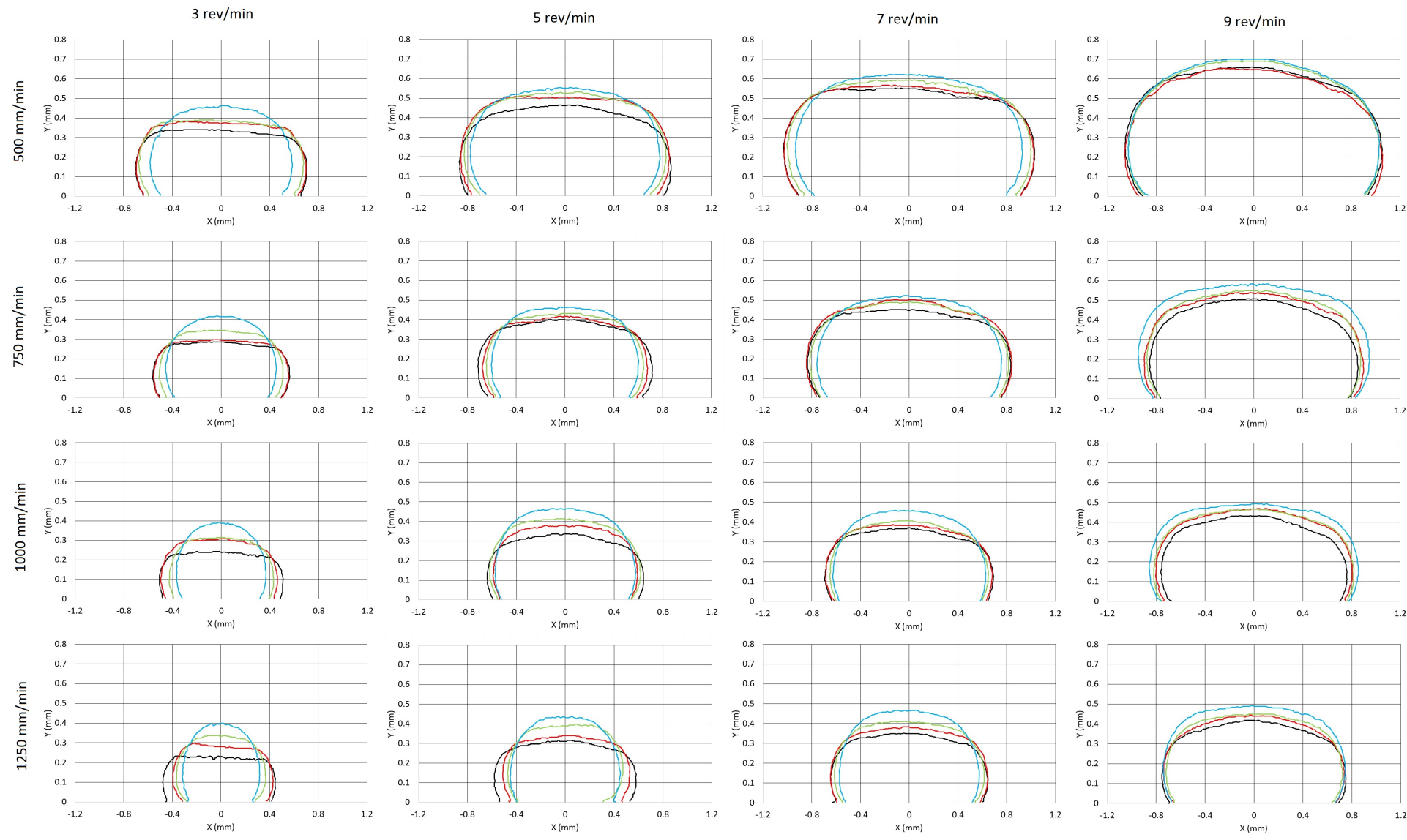

Figure 6: Contour maps of cross-sections of polymer depositions, PLA extruded at $220^{\circ} \mathrm{C}$, platform temperature $50^{\circ} \mathrm{C}$, grouped by filament feed velocity, $U_{0}$, and gantry velocity, $U_{P}$. Each colour represents a different nozzle gap height, $G_{N}$

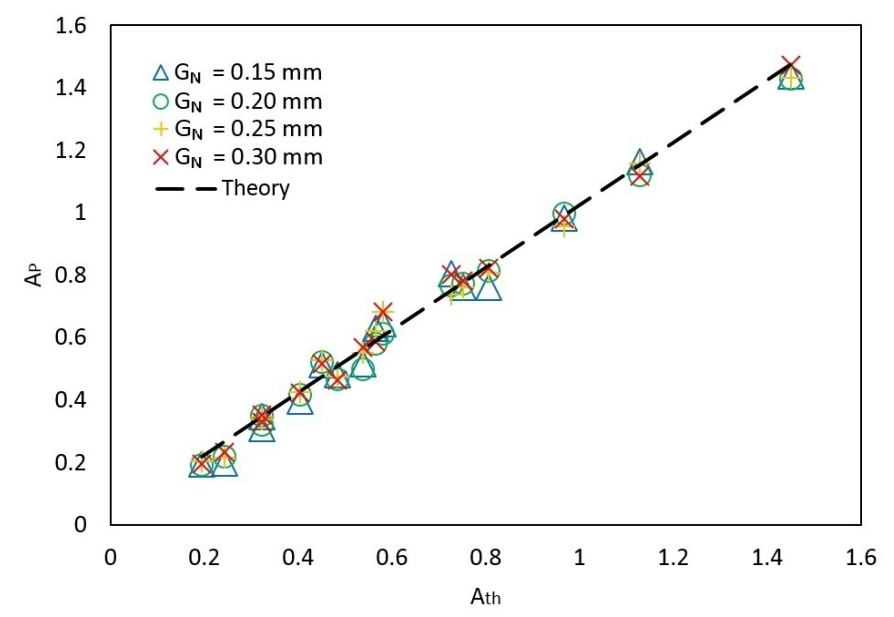

Figure 7: Theoretical area, $A_{P}$, compared to actual cross-sectional area, $A_{t h} \cdot G_{N}=0.2 m m, \mathrm{PLA}, 220^{\circ} \mathrm{C}$ extrusion temp, $50^{\circ} \mathrm{C}$ platform temp. The lines are given by Eq. 2 .

\subsection{Cross-sectional area}

We find that the cross-sectional area of a deposited filament can be predicted by a simple conservation of mass argument. That is;

$$
A_{P} U_{P}=A_{N} U_{N},
$$

where $A_{P}$ denotes the cross-sectional area of the deposited filament and

$$
A_{N}=\pi R_{N}^{2},
$$

is the cross-sectional area of the nozzle exit, with radius $R_{N}$. Thus, the cross-sectional area can be predicted by

$$
A_{P}=\pi R_{N}^{2} \frac{U_{N}}{U_{P}}
$$

as shown in Fig. 7; no material properties are required to make this prediction.

\subsection{Model for filament dimensions}

\section{Width of Filament, $W$}

The cross-section of a deposited filament is assumed to be elliptical, such that

$$
A_{P}=\pi \frac{W H}{4},
$$

where $W$ is the width and $H$ is the height of the filament. Making the assumption that $H=G_{N}$ (as in Ref. [23]), 


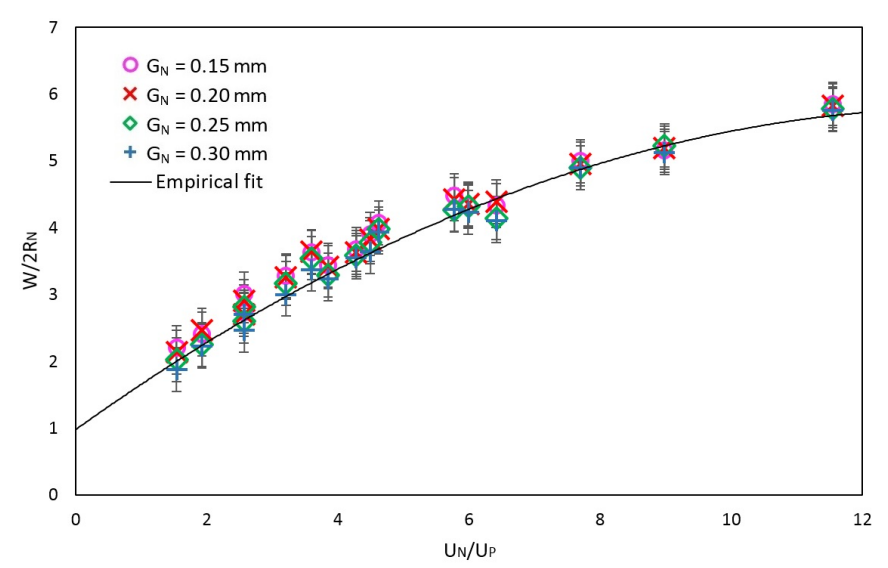

Figure 8: $W / 2 R_{N}$ plotted against $U_{N} / U_{P}$ for PLA material at $220^{\circ} \mathrm{C}$ extrusion temperature and $50^{\circ} \mathrm{C}$ platform temperature with nozzle gap sizes $G_{N}=0.15-0.3 \mathrm{~mm}$. The line is the empirical fit given by Eq. 7 with $\alpha=1.750$.

and equating Eqs. 4 and 5 gives a prediction for filament width, $W$, based on known parameters:

$$
W=4 \frac{R_{N}^{2}}{G_{N}} \frac{U_{N}}{U_{P}} .
$$

However, Eq. 6 fails to capture both experimental measurements and CFD simulations of the filament geometry [23], or capture the well document phenomenon of the filament height swelling above the nozzle gap size $\left(H>G_{N}\right)$ [21]. Consequently, we proceed by deriving an empirical form for the filament width, $W$, based on a convective cooling argument.

Fig. 8 shows the width of each cross section calculated from the CT images. We find that the measured width for each nozzle gap size, $G_{N}$, collapses to a master curve. The master curve can be described by

$$
\frac{W}{2 R_{N}}=\alpha \sqrt{\frac{U_{N}}{U_{P}}},
$$

where the pre-factor $\alpha$ is chosen to fit the data (via a nonlinear least-squares algorithm). This pre-factor is expected to depend on material properties, and a comparison with the value chosen for ABS is given in table 3.

\begin{tabular}{|l|l|}
\hline Material and temperatures & $\alpha$ \\
\hline Platform $50^{\circ} \mathrm{C}$ ABS $220^{\circ} \mathrm{C}$ & 1.252 \\
Platform $50^{\circ} \mathrm{C}$ PLA $220^{\circ} \mathrm{C}$ & 1.750 \\
Platform $60^{\circ} \mathrm{C}$ ABS $220^{\circ} \mathrm{C}$ & 1.245 \\
Platform $60^{\circ} \mathrm{C}$ PLA $220^{\circ} \mathrm{C}$ & 1.505 \\
\hline
\end{tabular}

Table 3: Table showing a list of materials with platform and extrusion temperatures with their respective $\alpha$ values.

Fig. 8 shows that the measured data is in quantitative agreement with Eq. 7 ; the width of a deposited filament $W$ is inversely proportional to $\sqrt{U_{P}}$.
A regression analysis was performed [33] on the dataset shown in Fig. 8. An $R^{2}$ value of 0.941 with an average standard error of 0.652 , which is presented as error bars in Fig. 8, showing that $94.1 \%$ of the variability in $W / 2 R_{N}$ can be attributed to $U_{N} / U_{P}$ and change in $G_{N}$. This shows statistical viability of Eqtn. 7 .

The choice of this functional form (Eq. 7) is motivated by the expectation that filament spreading is limited by convective cooling. That is $W \propto 1 / h_{f}$, where $h_{f}$ is the heat transfer coefficient for forced convection. For a plate moving in air, Lamberti et al. [34] find that $h_{f}$ proportional to the square root of the travel speed i.e. $h_{f} \propto \sqrt{U_{P}}$. Consequently, deposited filaments spread wider at slower speeds due to less cooling, whereas, fast print speeds induce less spreading due to faster convective cooling.

\section{Height of deposited filament}

Clearly we have seen that actual height of the deposited filament $H$ extends above the prescribed nozzle gap size $G_{N}$. In contrast to other work that assumes $H=G_{N}$, the derivation of an empirical form for $W$ (Eq. 7) allows us to make a prediction of this increased height by invoking conservation of mass.

Again by assuming the filament is elliptical and equating Eqs. 4 and 5, a prediction can be derived for the height:

$$
H=4 \frac{R_{N}^{2}}{W} \frac{U_{N}}{U_{P}} .
$$

Then, substituting in the empirical form for $W$ (Eq. 7) yields

$$
H=\frac{2 R_{N}}{\alpha} \sqrt{\frac{U_{N}}{U_{P}}} .
$$

(Note $\alpha$ is chosen by fitting to measurements of filament width in Eq. 7, and is expected to depend on material properties.)

It is often useful to present data in dimensionless form, particularly to highlight the ratio of expansion above the nozzle gap size. Thus, we can write

$$
\frac{H}{G_{N}} \propto \frac{2 R_{N}}{G_{N}} \sqrt{\frac{U_{N}}{U_{P}}},
$$

where $G_{N}$ is the set nozzle height. Fig. 9 shows Eq. 10 is in quantitative with the experimental data for a range of gap sizes. The regression analysis for the data presented in Fig. 9 showed an $R^{2}$ value of 0.847 in relation to Eqtn. 10 showing good statistical viability $(84.7 \%)$ of the model.

Fig. 10 shows the cross-sectional profile measured via CT-scanning compared to the model prediction. The $z(x)$ profile is generated by assuming the cross-section is a elliptical with minor axis $H / 2$ and major axis $W / 2$, as given by Eqs. 7 and 10. Then to give a more realistic shape, we assume that the contact line is pinned at $(x=-W / 2, z=$ $0),(x=W / 2, z=0)$. 


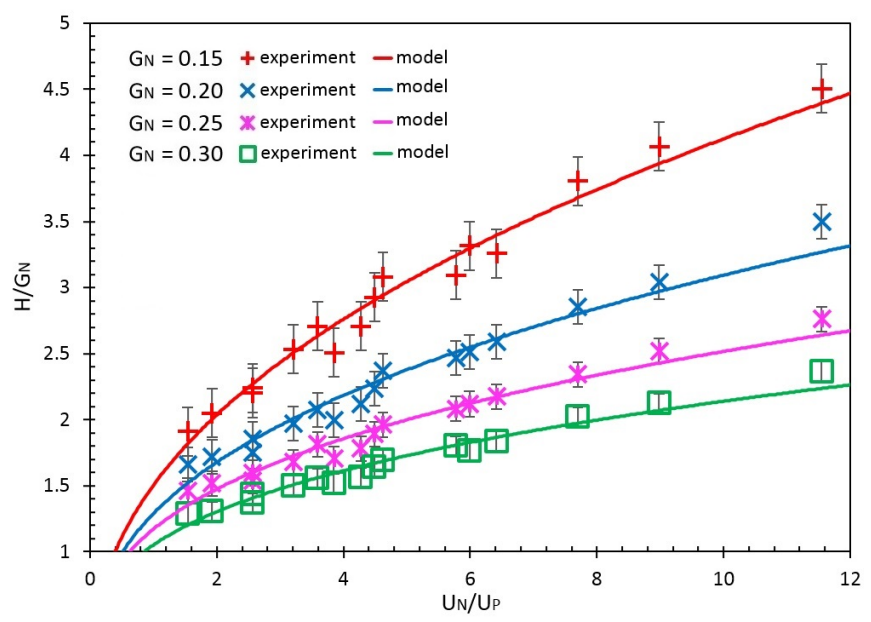

Figure 9: $H / G_{N}$ plotted against $U_{N} / U_{P}$ for PLA material at $220^{\circ} \mathrm{C}$ extrusion temperature and $50^{\circ} \mathrm{C}$ platform temperature with $G_{N}=$ $0.15-0.3 \mathrm{~mm}$. Lines are given by Eq. 10 with $\alpha=1.750$.

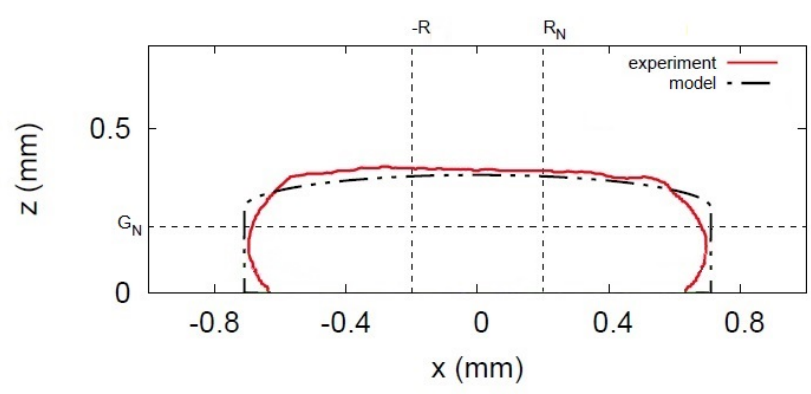

Figure 10: Cross-sectional profile, as measured using $C T$-scanning, compared to model prediction using $W$ and $H$ to generate an ellipse with a pinned contact line at $z=0$ (see text for details). Extrusion parameters are $U_{0}=3 \mathrm{rpm}$, where $1 \mathrm{rpm}=33.52 \mathrm{~mm} / \mathrm{s}$, $U_{P}=500 \mathrm{~mm} / \mathrm{s}, G_{N}=0.2 \mathrm{~mm}$.

\section{Discussion}

Fig. 6 reveals that there are many factors involved in forming a filament deposition of desired height and width, such as pairings of extrusion and travel velocities meaning setting parameters through trial and error can be insufficient in acquiring optimal working parameters.

\subsection{Controlling under/over extrusion}

Previous research has agreed that lower travel and extrusion velocities yield better build qualities within FFF processes, lower print temperatures can also help with the distortion of FFF depositions [35]. Zhou et al conclude in their 2017 paper on thermal behaviour of PLA in FFF; "reducing extrusion temperature, slowing printing speed, and decreasing layer thickness could help to reduce the vertical distortion and residual thermal stress" [36]. This obviously depends on the application, however, it is a broad rule that can be applied to the running of FFF apparatus.

Typical FFF tool-path generators give the user options to increase the rate of lay down thus decreasing the FFF

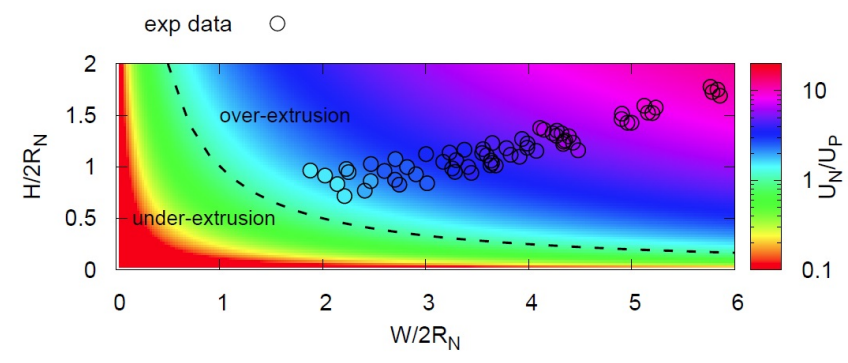

Figure 11: $W / 2 R_{N}$ plotted against $H / 2 R_{N}$ with $U_{N} / U_{P}$ represented by colour scale for all experimental datasets tested. Coloured areas are extrapolated for extended use in all dimensions with under and over-extrusion labelled by a dotted line.

process time, this is often done without explanation to the user and usually causes a decrease in quality of finished FFF parts. The data presented here shows that increasing print speed $U_{P}$, without changing $U_{N}$ accordingly, will result in undesired geometry, which is determined by conservation of mass and can be predicted by our model.

Fig. 11 shows how changing the ratio of the two speeds $U_{N} / U_{P}$ can give a vast range of filament geometries. The dashed line indicates a transition from under extrusion, where the filament cross-sectional area is less than the nozzle area, to over extrusion, where the filament area is greater than the nozzle size. The experimental data collected in this study is overlaid as filled circles. Thus, appropriate extrusion parameters can be chosen to give a desired filament geometry. This diagram is useful as a practical tool for designing toolpaths. For example, underextrusion can be exploited to generate finer filament resolutions, whereas over-extrusion can be exploited for gap filling, where required.

\subsection{ABS Filament Geometry}

Since conservation of mass is independent of material properties, the modelling approach presented above can be applied to any printing material. It particular, we have used to the same experiment to investigate the track dimensions of printed ABS. We test the applicability of the generic equation for conservation of mass (Eq. 3), which is material independent, together with Eqs. 7 and 10 for $W$ and $H$, where the material dependence is currently unknown. The full data set is presented in the Appendix

In particular, Fig. A.1 shows how Eq. 4 also accurately predicts the cross-sectional area of an ABS filament. The greater spread of data from the conservation of mass theory, particularly for larger $A_{P} / A_{N}$ ratios, demonstrates that $\mathrm{ABS}$ is less reliable than PLA. Regression analyses on both PLA and ABS $A_{P} / A_{N}$ datasets shows $99.0 \%$ and $96.6 \%$ variability. Thus, ABS is slightly less predictable.

It is well known that ABS is more difficult to use in FFF than PLA [37] [38]; the semi-crystalline regions found in PLA tend to give better structural integrity than purely amorphous ABS, which tends to flow for longer periods of 
time. This effect can also lead to curving, or banding, of the layer - a common problem in ABS parts [39].

Fig. 12 shows that the empirical form given by Eq.7 is also consistent with ABS material properties; in fact only a small shift of Eq. 7 is required to account for the ABS data. In particular, Table 3 details the pre-factor $\alpha$ required to best fit the $\mathrm{ABS}$ data.

Since ABS and PLA are very different polymers, this behaviour suggests that the underlying mechanism for spreading is due to external printing conditions rather than inherent characteristics of the polymer itself, and that a small dependence on material properties enters the unknown pre-factor $\alpha$ in Eq. 7 .

Furthermore, we observe that PLA spreads further in the $x$-direction compared to ABS at the same print ratio. This is in contrast to our initial intuition that semicrystalline polymers would demonstrate smaller filament widths compared to amorphous polymers; semi-crystalline polymers are known to shrink during crystallisation due to a change in volume [37]. The effect we observe is actually a result of the chosen bed temperature. PLA has a much lower glass transition than $\mathrm{ABS}\left(60^{\circ} \mathrm{C}\right.$ compared to $110^{\circ} \mathrm{C}$ ), thus has significantly more time to flow to larger filament widths.

The data presents a spectrum of FFF parameters available to typical FFF machines [29], although we have yet to test the effect of nozzle size, and demonstrates that the model can be applied to both PLA and ABS - two most popular materials used within FFF. We expect the model can also be applied to other polymers, by simply adjusting the pre-factor, $\alpha$, in Eq. 7.

Clearly further research into the complex expansion (and contraction) process that occurs during deposition is required for a complete theory. Warping and de-bonding of materials from the build surface may also need to be investigated but are outside the scope of this paper. Specialist polymers could also create other phenomenons, such as fibre filled materials and polymer/rubber blends different flow properties. Nevertheless, with only a single calibration, this simple model can give fast geometrical predictions over a reasonably wide range of extrusion parameters. Thus, we expect the model to be useful for anybody writing tool-paths or software for FFF apparatus.

\subsection{Build plate temperature}

Heated build platforms and build chambers are commonly used in FFF setups for more even distribution and dissipation of thermal energy throughout the material. Here we have tested the effect of two build plate temperature $T_{a}=50$ and $60^{\circ} \mathrm{C}$ on the track dimensions. In particular, Fig. 13 shows the final width of a PLA filament for increasing print speed ratio.

It was expected that a higher build plate temperature would extend the time spent above the glass transition temperature and therefore allow the material to flow further. However, counter-intuitively, we find that a lower build plate temperature leads to larger filament widths,

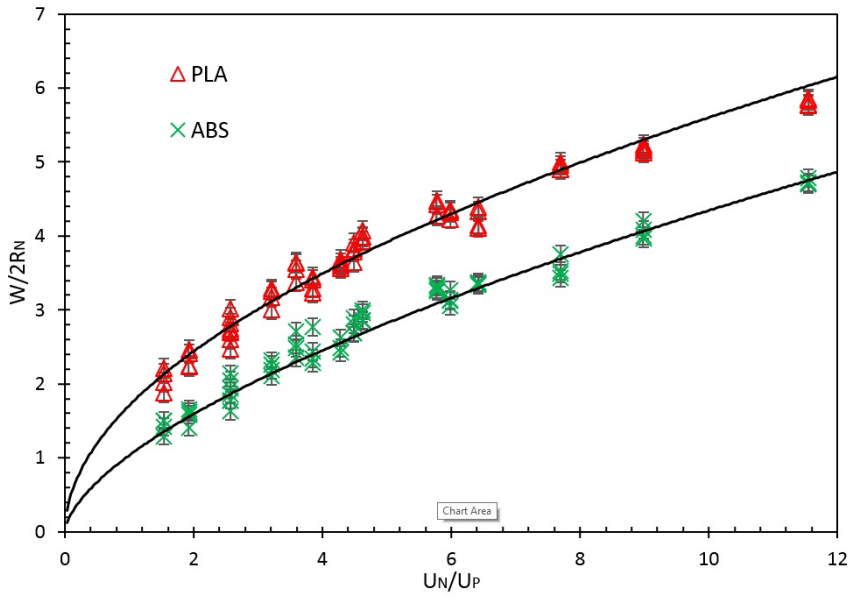

Figure 12: $U_{N} / U_{P}$ plotted against $W / 2 R_{N}$ for PLA and ABS materials at $220^{\circ} \mathrm{C}$ extrusion temperature and $50^{\circ} \mathrm{C}$ platform temperatures. The lines are given by Eq. 7 for all $G_{N}$ values with $\alpha=1.750$ and 1.252 respectively.

$W$. We believe this effect to be due to how well the polymer wets to the build plate at different temperatures i.e. wetting favours the lower build plate temperature, resulting in larger filament widths. This effect is also contained in pre-factor $\alpha$.

Controlling the build plate temperature can evidently be used to control build geometry, as well as inter-diffusive welding dynamics. Better understanding of this effect could lead to new routes to superior finishing results.

\subsection{First layer effects}

The heights to which the polymer depositions expand gives us an indicator of both the process and the materials limitations in terms of how much volume can be extruded at once. Gaining unnecessary layer height within the FFF build process can cause the printed objects height to exceed that of the nozzle height in later stages of the build process, this can cause a lack of free space for polymer to leave the nozzle and eventually cause blockages and damage to the apparatus, mainly the nozzle tip.

Since the height of a deposited filament exceeds the nozzle gap size, most slicing software uses a function which leaves an added gap in the $\mathrm{z}$-direction after the initial layer, however, other applications may be necessary which step away from the conformities of standard FFF practises. These include the preparation of single-layered products which require the precision of FFF but not necessarily its full three-dimensional qualities. It is therefore logical that by using the equations presented, new applications could be found, such as preparation of antenna within FFF communications devices which are a large source of research in the field of electrical and medical engineering [40, 41]. Staying with the typical FFF process, however, using the supplied data it is now possible to predict with a reasonable degree of accuracy, the initial extrusions on glass build 
plates with a known height and width from which can be extrapolated the correct spacing between both depositions and layers. This could eliminate the need for correctional values commonly used during the FFF process to tweak the parameters visually on the fly.

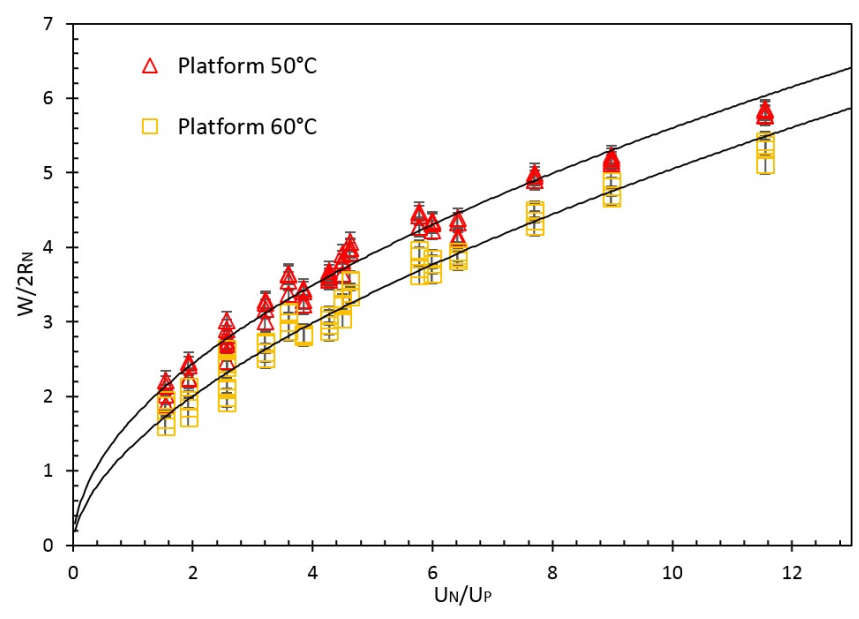

Figure 13: $U_{N} / U_{P}$ plotted against $W / 2 R_{N}$ for PLA material at $220^{\circ} \mathrm{C}$ extrusion temperature and both $50^{\circ} \mathrm{C}$ and $60^{\circ} \mathrm{C}$ platform temperatures. The lines are given by Eq. 7 for all $G_{N}$ values. $\alpha=1.750$ and 1.505 respectively.

\section{Concluding Remarks}

This paper presents a simple model based on conservation of mass to predict the height, width and crosssectional area of polymer depositions extruded onto a glass surface using FFF. This proposed model simplifies full CFD calculations [23], moves away from the assumption that $H=G_{N}$, and implicitly accounts for cooling effects via application of an empirical equation. In this way, we are able to capture expansion of the filament above the nozzle gap size; a first-layer effect commonly seen in FFF and readily observed in our CT scanning images.

Subsequent layers after the initial lay down procedures in FFF may not encounter these first-layer effects presented here, as the surface the polymer is to be deposited on to is no longer permanently solid and is in fact a textured, melted polymer itself. Thus, by applying the assumption $H=G_{N}$, the model can be used to directly predict the width of subsequently deposited filaments (via Eq. 6).

The model can be used to predict track dimensions for a wide range of nozzle gap sizes and velocity ratios, and will allow FFF-users to design tool-paths more effectively. In particular, the appropriate $U_{N}$ and $U_{P}$ can be selected to achieve the height and width required to fill an area of space. This facilitates the calculation of the optimum filament spacing to optimise the contact area between adjacent filaments, which in turn will increase the strength of components - a feature which FFF apparatus and filament manufacturers are always trying to improve. On the other hand, the model can be used to exploit under extrusion and create finer resolutions.

Furthermore, to avoid CFD simulation, the deposition model of McIlroy et al. [42] relies on prior knowledge of the deposition shape in order to investigate micro-structural properties at the weld line. For example, the deposition shape is found to affect the polymer deformation and consequently inter-diffusion dynamics [43] and crystallization kinetics [44]. Consequently, the study presented here is essential to the development of simple molecularly-aware models that do not require full CFD calculations.

FFF manufactured PLA and ABS are viscoelastic and therefore demonstrate die swell, as well as flow and temperaturedependent behaviour. Understanding this behaviour is key to developing a more complete theory, in particular knowledge of the pre-factor $\alpha$ in Eq. 7, and will be the focus of our future work.

\section{References}

[1] K. P. Motaparti, G. Taylor, M. C. Leu, K. Chandrashekhara, J. Castle, M. Matlack, Experimental investigation of effects of build parameters on flexural properties in fused deposition modelling parts, Virtual and Physical Prototyping 12 (2017) $207-220$.

[2] J. W. Stansbury, M. J. Idacavage, 3d printing with polymers: Challenges among expanding options and opportunities, Dental Materials 32 (2016) 54-64.

[3] C. K. Chua, K. F. Leong, 3D Printing and Additive Manufacturing: Principles and Applications (with Companion Media Pack) of Rapid Prototyping Fourth Edition, World Scientific Publishing Company, 2014.

[4] A. Bandyopadhyay, S. Bose, Additive manufacturing, CRC Press, 2015.

[5] Q. Dao, J. C. Frimodig, H. N. Le, X.-Z. Li, S. B. Putnam, K. Golda, J. Foyos, R. Noorani, B. Fritz, Calculation of shrinkage compensation factors for rapid prototyping (fdm 1650), Computer Applications in engineering education 7 (1999) 186195.

[6] C. W. Ziemian, R. D. Ziemian, K. V. Haile, Characterization of stiffness degradation caused by fatigue damage of additive manufactured parts, Materials \& Design 109 (2016) 209-218.

[7] P.-T. Lan, S.-Y. Chou, L.-L. Chen, D. Gemmill, Determining fabrication orientations for rapid prototyping with stereolithography apparatus, Computer-Aided Design 29 (1997) 53-62.

[8] J. Hur, K. Lee, The development of a cad environment to determine the preferred build-up direction for layered manufacturing, The International Journal of Advanced Manufacturing Technology 14 (1998) 247-254.

[9] O. Es-Said, J. Foyos, R. Noorani, M. Mendelson, R. Marloth, B. Pregger, Effect of layer orientation on mechanical properties of rapid prototyped samples, Materials and Manufacturing Processes 15 (2000) 107-122.

[10] O. A. Mohamed, S. H. Masood, J. L. Bhowmik, Optimization of fused deposition modeling process parameters: a review of current research and future prospects, Advances in Manufacturing 3 (2015) 42-53.

[11] J. Wang, H. Xie, Z. Weng, T. Senthil, L. Wu, A novel approach to improve mechanical properties of parts fabricated by fused deposition modeling, Materials \& Design 105 (2016) 152-159.

[12] H. W. Guan, M. M. Savalani, I. Gibson, O. Diegel, Influence of fill gap on flexural strength of parts fabricated by curved layer fused deposition modeling, Procedia Technology 20 (2015) 243248. 
[13] S. Ziemian, M. Okwara, C. W. Ziemian, Tensile and fatigue behavior of layered acrylonitrile butadiene styrene, Rapid Prototyping Journal 21 (2015) 270-278.

[14] T.-M. Wang, J.-T. Xi, Y. Jin, A model research for prototype warp deformation in the fdm process, The International Journal of Advanced Manufacturing Technology 33 (2007) 1087-1096.

[15] B. N. Turner, R. Strong, S. A. Gold, A review of melt extrusion additive manufacturing processes: I. process design and modeling, Rapid Prototyping Journal 20 (2014) 192-204.

[16] M. K. Agarwala, V. R. Jamalabad, N. A. Langrana, A. Safari, P. J. Whalen, S. C. Danforth, Structural quality of parts processed by fused deposition, Rapid prototyping journal 2 (1996) $4-19$.

[17] A. Bellini, S. Guceri, M. Bertoldi, Liquefier dynamics in fused deposition, Journal of Manufacturing Science and Engineering 126 (2004) 237-246.

[18] M. AtifYardimci, T. Hattori, S. I. Guceri, S. C. Danforth, Thermal analysis of fused deposition, in: 1997 International Solid Freeform Fabrication Symposium, 1997.

[19] N. Venkataraman, S. Rangarajan, M. Matthewson, B. Harper, A. Safari, S. Danforth, G. Wu, N. Langrana, S. Guceri, A. Yardimci, Feedstock material property-process relationships in fused deposition of ceramics (fdc), Rapid Prototyping Journal 6 (2000) 244-253.

[20] R. S. Crockett, The liquid-to-solid transition in stereodeposition techniques (1997).

[21] A. Bellini, Fused deposition of ceramics: a comprehensive experimental, analytical and computational study of material behavior, fabrication process and equipment design, 2002.

[22] A. Gleadall, I. Ashcroft, J. Segal, Volco: A predictive model for 3d printed microarchitecture, Additive Manufacturing (2018).

[23] M. P. Serdeczny, R. Comminal, D. B. Pedersen, J. Spangenberg, Experimental validation of a numerica model for the strand shape in material extrusion additive manufacturing, Additive Manufacturing (2018). doi:https://doi.org/10.1016/j.addma.2018.09.022.

[24] M. Mirzaee, S. Noghanian, L. Wiest, I. Chang, Developing flexible 3d printed antenna using conductive abs materials, in: Antennas and Propagation \& USNC/URSI National Radio Science Meeting, 2015 IEEE International Symposium on, IEEE, 2015, pp. 1308-1309.

25] C. B. Pinnock, E. M. Meier, N. N. Joshi, B. Wu, M. T. Lam Customizable engineered blood vessels using $3 \mathrm{~d}$ printed inserts, Methods 99 (2016) 20-27.

[26] S. J. Hollister, Porous scaffold design for tissue engineering, Nature materials 4 (2005) 518.

[27] J. Tu, G. H. Yeoh, C. Liu, Computational fluid dynamics: a practical approach, Butterworth-Heinemann, 2018.

[28] P. G. Lafleur, B. Vergnes, Polymer extrusion, John Wiley \& Sons, 2014.

[29] J. Go, S. N. Schiffres, A. G. Stevens, A. J. Hart, Rate limits of additive manufacturing by fused filament fabrication and guidelines for high-throughput system design, Additive Manufacturing 16 (2017) 1-11.

[30] R. Griskey, Polymer process engineering, Springer Science \& Business Media, 2012.

[31] F. Xu, H. Loh, Y. Wong, Considerations and selection of optimal orientation for different rapid prototyping systems, Rapid Prototyping Journal 5 (1999) 54-60.

[32] B. Wittbrodt, J. M. Pearce, The effects of pla color on material properties of 3-d printed components, Additive Manufacturing 8 (2015) 110-116.

[33] N. R. Draper, H. Smith, Applied regression analysis, volume 326, John Wiley \& Sons, 2014.

[34] G. Lamberti, G. Titomanlio, V. Brucato, Measurement and modelling of the film casting process 1 . width distribution along draw direction, Chemical Engineering Science 56 (2001) 57495761 .

[35] Y. Zhou, T. Nyberg, G. Xiong, D. Liu, Temperature analysis in the fused deposition modeling process, in: Information Science and Control Engineering (ICISCE), 2016 3rd International
Conference on, IEEE, 2016, pp. 678-682.

[36] X. Zhou, S.-J. Hsieh, Y. Sun, Experimental and numerical investigation of the thermal behaviour of polylactic acid during the fused deposition process, Virtual and Physical Prototyping 12 (2017) 221-233.

[37] J. M. Chalmers, R. J. Meier, Polymer degradation and oxidation: An introduction, Comprehensive Analytical Chemistry 53 (2008) 387-450

[38] H. F. Giles Jr, E. M. Mount III, J. R. Wagner Jr, Extrusion: the definitive processing guide and handbook, William Andrew, 2004

[39] M. Alsoufi, A. El-Sayed, Warping deformation of desktop 3d printed parts manufactured by open source fused deposition modeling (fdm) system 17 (2017) 7-16.

[40] D. A. Cadman, S. Zhang, J. Vardaxoglou, Fused deposition modelling for microwave circuits \& antennas (2016).

41] P. I. Deffenbaugh, R. C. Rumpf, K. H. Church, Broadband microwave frequency characterization of 3-d printed materials, IEEE Transactions on Components, Packaging and Manufacturing Technology 3 (2013) 2147-2155.

[42] C. McIlroy, P. D. Olmsted, Deformation of an amorphous polymer during the fused-filament-fabrication method for additive manufacturing, Journal of Rheology 61 (2017) 379-397.

[43] C. McIlroy, P. Olmsted, Disentanglement effects on welding behaviour of polymer melts during the fused-filament-fabrication method for additive manufacturing, Polymer 123 (2017) 376391.

[44] C. McIlroy, R. S. Graham, Modelling flow-induced crystallisation during fused filament fabrication of semi-crystalline polymer melts, Additive Manufacturing accepted (????). 


\section{Appendix A. Additional ABS data}

Here is included additional data for ABS material associated with the experiments presented. This data was been processed in the same manner as the PLA datasets and is included as proof of Model Generality as mentioned in section 4 .

Fig. A.1 shows the area calculations given by Eq. 4 and is the ABS equivalent of Fig. 7. Fig. A.2 shows the area calculations given by Eq. 10 and is the ABS equivalent of Fig. 9. Contour maps of the ABS depisition cross-sections in Fig. A.3 are also included as a visual comparison to the PLA equivelant in Fig. 6.

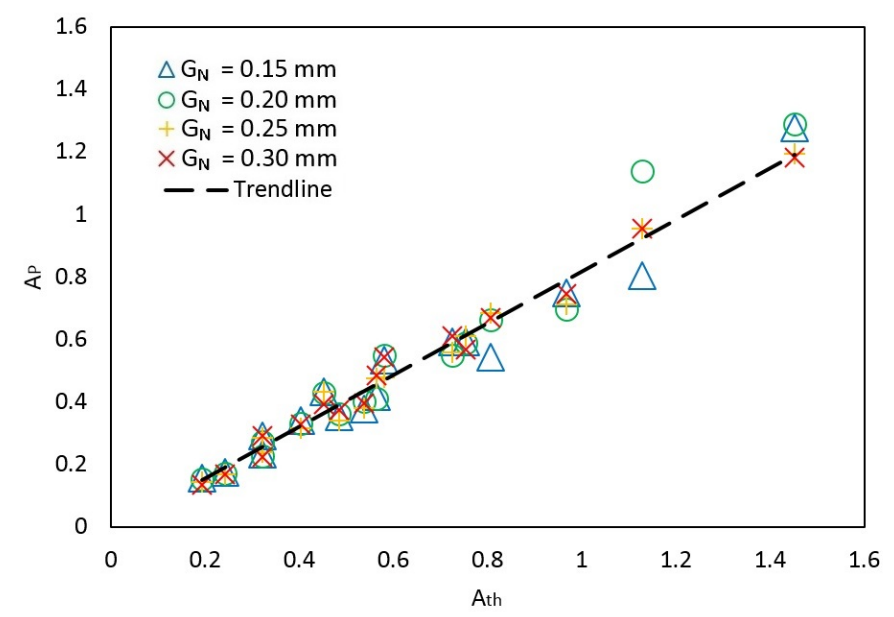

Figure A.1: Theoretical area, $A_{P}$, compared to actual cross-sectional area, $A_{t h} . G_{N}=0.2 \mathrm{~mm}, \mathrm{ABS}, 220^{\circ} \mathrm{C}$ extrusion temp, $50^{\circ} \mathrm{C}$ platform temp. The lines are given by Eq. 4.

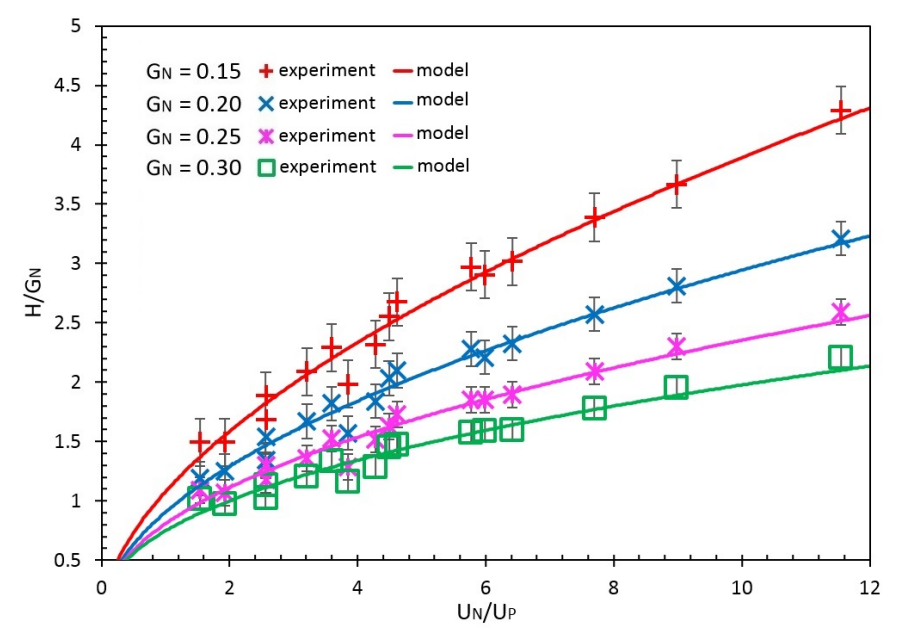

Figure A.2: $U_{N} / U_{P}$ plotted against $H / G_{N}$ for ABS material at $220^{\circ} \mathrm{C}$ extrusion temperature and $50^{\circ} \mathrm{C}$ platform temperature at $G_{N}=0.15-0.3 m m$. The lines are given by Eq. 10 with $\alpha=1.252$. 

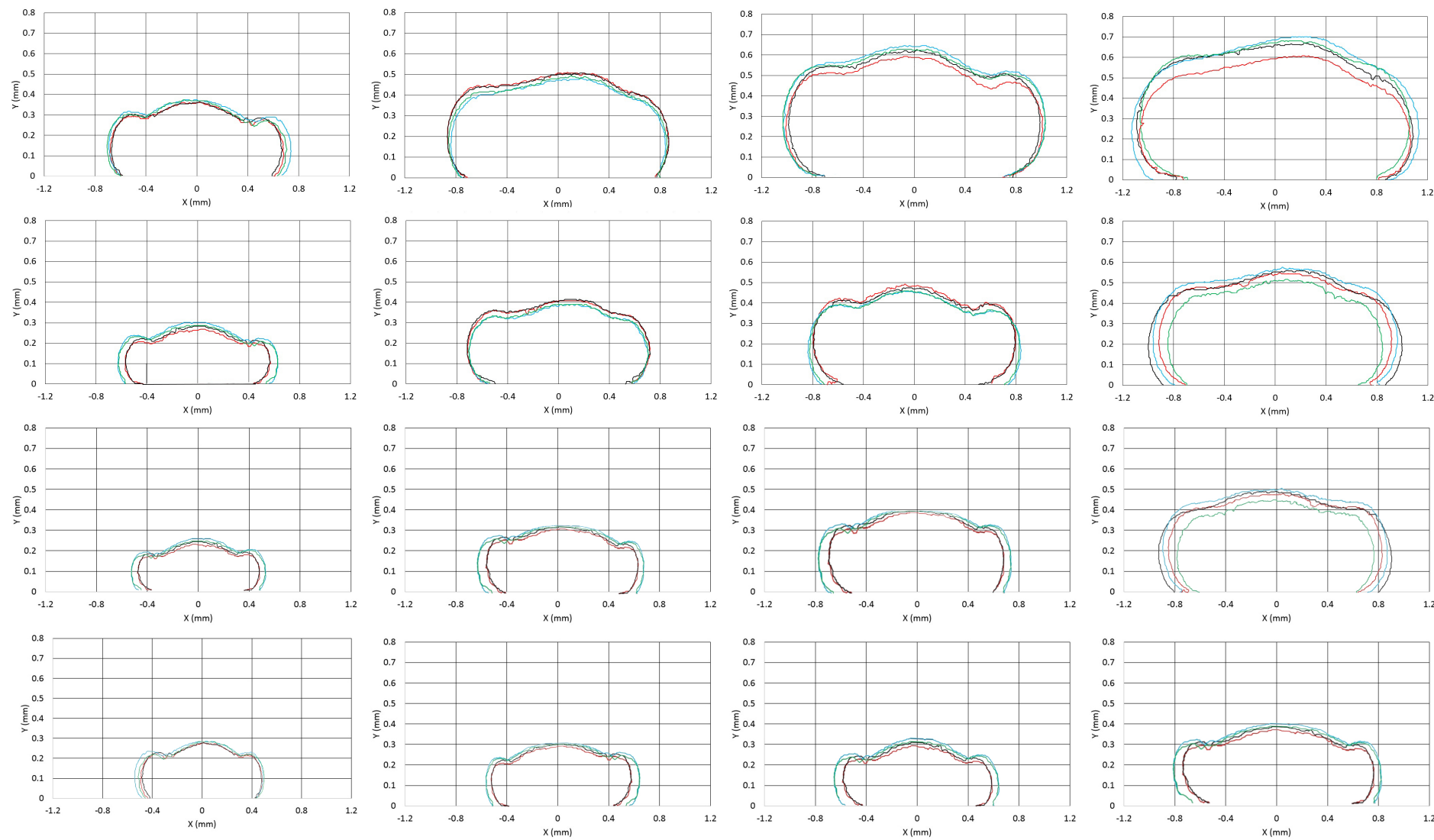

Figure A.3: Contour maps of cross-sections of polymer depositions, ABS extruded at $220^{\circ} \mathrm{C}$, platform temperature $50^{\circ} \mathrm{C}$, grouped by filament feed velocity, $U_{0}$, and gantry velocity, $U_{P}$. Each colour represents a different nozzle gap height, $G_{N}$ 